American Journal of Applied Sciences 6 (4): 784-787, 2009

ISSN 1546-9239

(C) 2009 Science Publications

\title{
Gravity Assist and Scattering off AGB Stars off Molecular Clouds
}

\author{
Ahmad Hashem Abdelhadi \\ College of Arts and Sciences, \\ New York Institute of Technology, P.O. Box 840878, Amman 11184, Jordan
}

\begin{abstract}
The scattering of stellar orbits by galactic molecular clouds is studied in hope of explaining isotopic peculiarities of presolar grains from Asymptotic Giant Branch (AGB) stars. Silicon isotopic anomalies found in the mainstream Silicon Carbide $(\mathrm{SiC})$ grains are observed to have heavy isotopes enriched. To explain the isotopic heaviness I propose that AGB stars that formed in the interior region of the galaxy, with metallicity higher than solar, change their orbits during close encounters with molecular clouds. The AGB stars give up their SiC grains in winds deposited in the solar neighborhood prior to the birth of the Sun. I model the dynamics by superposing on a standard axisymmetric galactic potential the discrete potentials of thousands of molecular clouds moving in constant circular orbits. Gravity assist occurs when a star scattered into a crossing orbit by one cloud experiences forward scattering from a second cloud.
\end{abstract}

Key words: Silicon carbide, AGB stars, gravity assist

\section{INTRODUCTION}

Many studies (e.g., 5 and 14) have shown that the mainstream presolar Silicon Carbide $(\mathrm{SiC})$ grains extracted from meteorites formed in asymptotic giant branch stars. Ion probe studies show them to have ${ }^{29} \mathrm{Si} /{ }^{28} \mathrm{Si}$ and ${ }^{30} \mathrm{Si} /{ }^{28} \mathrm{Si}$ isotopic ratios larger than those found in solar material (Fig. 1). The problem lies in the fact that the mainstream presolar $\mathrm{SiC}$ grains are richer in the heavier silicon isotopes than the solar composition even though the grains must have originated in stars that formed prior to the Sun. To deliver their grains to the solar birth cloud, the donor stars clearly must have been born and evolved prior to solar birth. Homogeneous chemical evolution of these $\mathrm{Si}$ isotope ratios increase monotonically with time and simply can not accommodate earlier stars having higher ratios. Therefore, one seeks reasons for the high isotopic ratios of the majority of donor stars. Clayton ${ }^{3}$ argued that this silicon isotopic richness is due in part to the diffusion of the parent AGB stars from more central birthplaces within the Galaxy. Due to a more evolved interior position, these central birthplaces are known to have a higher metallicity. The outward diffusion can be likened to the gravity assist used in solar system exploration. AGB stars scatter by near gravitational encounters with molecular clouds. This changes the orbits of some to spend much time at larger galactic radii than those of their birth. There they give out their stardust plot.

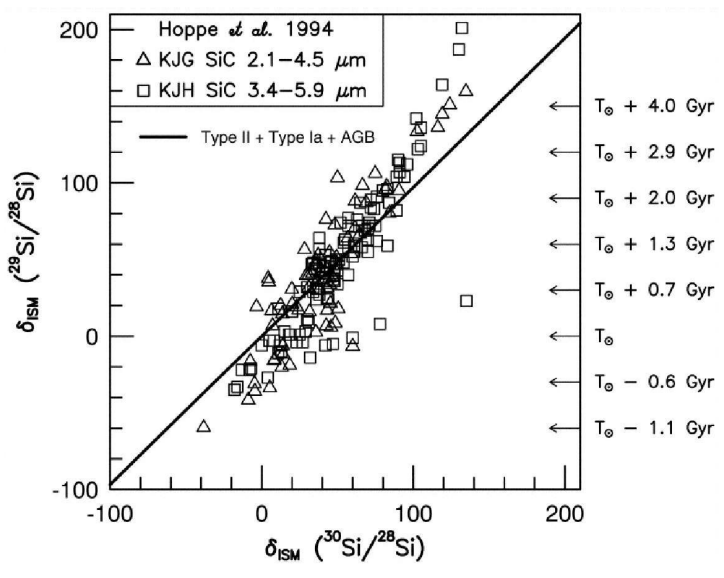

Fig. 1: Silicon isotope deviations in a three-isotope

Murchison SiC samples measured by Hoppe et al. are shown and have a best-fit slope of $4 / 3$. The renormalized mean Interstellar Medium (ISM) evolution is shown as the solid line and by construction it passes through solar abundances at $\mathrm{t}=\mathrm{t}_{\text {solar }}$. With such a construction, deviations with respect to solar abundances and with respect to interstellar abundances are the same $\delta_{\text {solar }}=\delta_{\mathrm{ISM}^{[3]}}$.

\section{SCATTERING OFF AGB STARS}

Since I propose to deal with star-molecular-cloud encounters, one might ask if stars collide with one

Corresponding Author: $\quad$ Ahmad H Abdelhadi, College of Arts and Sciences, New York Institute of Technology, PO Box 840878, Amman 11184, Jordan, Tel: +962-6471-1322, Fax: +962-6471-1310, 
another. This que has been addressed by other researchers (e.g., 2 and 11) and the interval between collisions was calculated to be $10^{19}$ years. This is considerably longer than the believed age of the Galaxy (years). Evidently, the stars in the Galaxy do not suffer significant individual deflections due to the presence of other stars and large-angle collisions between stars are very infrequent and do not contribute to migration of AGB stars.

To calculate the orbits of stars requires a galactic mass model that defines an expression for the galactic gravitational potential. In using such a model, I neglect the forces from individual stars and consider only the large-scale forces from the overall mass distribution, which is made up of thousands of millions of stars. This takes the force fields of the galaxy to be smooth, neglecting small-scale irregularities due to individual stars or larger objects like globular clusters. Hence, I use a numerically convenient axisymmetric gravitational potential and density distribution proposed by Miyamoto and $\mathrm{Nagai}^{6}$ in 1975 :

$$
\Phi(\mathrm{R}, \mathrm{z})=-\sum_{\mathrm{i}=1}^{3} \frac{\mathrm{GM}_{\mathrm{i}}}{\sqrt{\mathrm{R}^{2}+\left(\mathrm{a}_{\mathrm{i}}+\sqrt{\mathrm{z}^{2}+\mathrm{b}_{\mathrm{i}}^{2}}\right)^{2}}}
$$

Where:

$\mathrm{R}=$ The galactocentric radius

$\mathrm{z}=$ The height above and below the galactic midplane

Respectively, the three parameters a (in $\mathrm{kpc}$ ), b (in kpc) and $\mathrm{M}$ (in $10^{10} \mathrm{M}_{\text {Solar }}$ ) are: 0.0, 0.3 and 1.8 for the bulge; $6.2,0.4$ and 17.4 for the disk; 0.0, 31.2 and 83.5 for the corona ${ }^{[4]}$. Thus, depending on the choice of the two parameters a and b, $\Phi$ can represent the potential of anything from an infinitesimally thin disk to a spherical one. This expression is free from singularities everywhere in space and is differentiable an unlimited number of times with respect to the space coordinates $^{[6]}$. It also tends to the Newtonian potential for a point mass when $\mathrm{R}$ and $\mathrm{z}$ become large. I should also mention that I omit non-axisymmetric structures such as an inner bar, the spiral arms and local features such as Gould's Belt ${ }^{[3]}$. However, this potential is in good agreement with observational determinations of the Galactic rotational velocity curve ${ }^{[15]}$, the vertical force $\mathrm{K}_{\mathrm{z}}$ at the Sun's position and the total local mass density $^{[5]}\left(\rho_{\text {solar }} \sim 0.15 \mathrm{M}_{\text {solar }} \mathrm{pc}^{-3}\right)$. Molecular clouds individually produce fluctuations in the gravitational field seen by nearby stars, which in return produce changes in the magnitude and direction of each stellar velocity. A typical star's orbit is shown in Fig. 2.

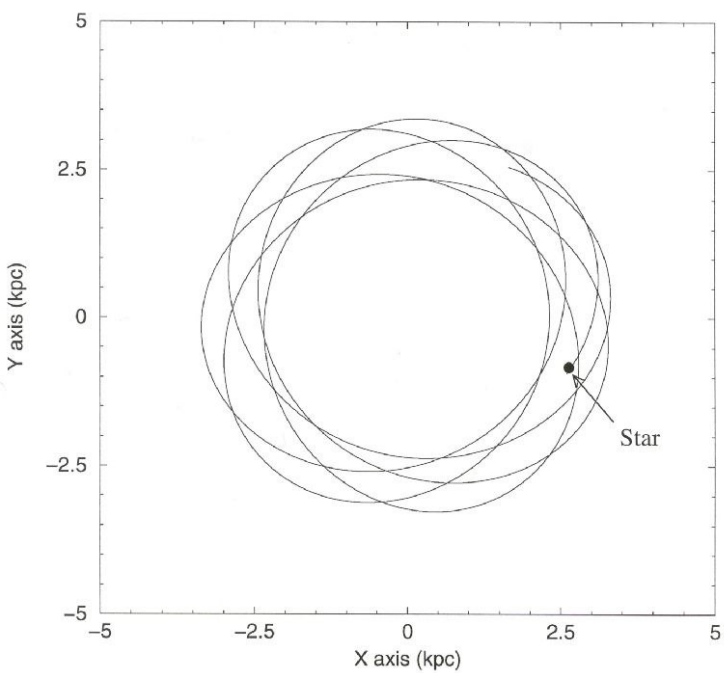

Fig. 2: A face-on galactic plane orbit that forms a rosette. The star has an initial circular velocity $196.2 \mathrm{~km} \mathrm{sec}^{-1}$ and lived for 0.5 billion years

Molecular clouds are detected and mapped by observing millimeter emission from a trace molecule: Carbon monoxide $(\mathrm{CO})^{[8]}$. I used the results from the different galactic plane surveys ${ }^{[8,10]}$ to distribute the molecular clouds radially. These results show that the molecular clouds are the dominant component of the interstellar medium in the inner half of the disk at $\mathrm{R}<0.8 \mathrm{R}_{\text {solar }}$ with a rise between $\mathrm{R}=5$ and $\mathrm{R}=7 \mathrm{kpc}$ and a sharp fall toward the larger radius where there is very little emission beyond $\mathrm{R}=15 \mathrm{kpc}$. Most of the surveys ${ }^{[1,8,10,12]}$ suggest a median cloud mass of about $10^{5} \mathrm{M}_{\text {Solar }}$.

Since my problem concerns the effect of the molecular clouds on the motion of stars through an otherwise azimuthally symmetric potential, I can utilize a simple approximation of their masses and the radial distribution for that purpose. And to a first approximation I can take the molecular clouds to be spherical. It's not my intention to model neither the molecular clouds nor their radial distribution. The goal is not to study molecular clouds themselves but rather their cumulative perturbations of the stellar orbits in an azimuthally symmetric potential. The goal is to study the extent to which the radial position of stars that were born on circular orbits will be radially displaced from their circular orbits at fixed stellar evolutions times. For that purpose I employ a Gaussian distribution that randomly generates positions for the molecular clouds along the galactic mid plane. The transformation I use for the Gaussian deviations is ${ }^{[7]}$ : 


$$
\mathrm{R}=\sqrt{-2 \ln \mathrm{a}_{1}} \cos 2 \pi \mathrm{a}_{2}
$$

where, $\mathrm{R}$ is the galactocentric radius, $\mathrm{a}_{1}$ and $\mathrm{a}_{2}$ are two uniform deviates on $(0,1)$. The Gaussian distribution presented here is not "fully" axisymmetric nor smooth because clouds are distributed randomly; meaning strings of high dense areas of molecular clouds are instituted and placed randomly to account for the observed fluctuation in the radial distribution.

A typical calculation is set up to trace $10^{4}$ molecular clouds and $10^{3}$ stars for two billion years. I place the molecular clouds randomly on circular orbits in the galactic mid plane. The clouds do not have peculiar velocities thus their motions do carry them in the z-direction and their circular orbits are not perturbed, i.e., they do not oscillate radially nor azimuthally. Each cloud has mass $10^{5} \mathrm{M}_{\text {solar }}$ and $10 \mathrm{pc}$ radius. Since each molecular cloud is more massive than the star; a gravitational force from a nearby star on the molecular cloud is going to be small and thus neglected. To a first approximation the molecular clouds retain their circular orbits and constant angular momentum. The stars are initially distributed randomly in radial zones. A zone is a ring with $500 \mathrm{pc}$ width; for example, the region between $\mathrm{R}=4.0 \mathrm{kpc}$ and $\mathrm{R}=4.5$ $\mathrm{kpc}$ is one zone. Each zone has $10^{3}$ stars positioned randomly in $\mathrm{R}$ and in its azimuthal angle in the galactic mid plane. Each star is given a randomly generated peculiar velocity between 10 and $20 \mathrm{~km} / \mathrm{s}$. The direction of the peculiar velocity is also randomly chosen in $\theta$ and $\phi$, where $\theta$ and $\phi$ are the known spherical coordinates. Added to the peculiar velocity is the proper circular velocity deduced from Miyamoto and Nagai potential. With these initial conditions the system evolves for two billion years. Both molecular clouds and stars maintain their shapes and masses throughout collisions and calculations. I have used a numerical method called the Bulirsch-Stoer algorithm ${ }^{[7]}$ with adaptive time step to trace the orbits of stars by integrating the equation of motion for the stars. The nearby molecular clouds' gravitational field mainly governs a star's trajectory. The total force F (per unit mass) on a star is the sum of the galactic gravitational force derived from Miyamoto and Nagai potential and the nearby molecular-cloud gravitational forces. Nearby molecular clouds defined as ones found within a cube of $500 \mathrm{pc}$ from a star at its center. Molecular clouds outside the cube are not included specifically in the total force at that particular point of time. The distant clouds are assumed part of the azimuthal galactic potential.

\section{DISCUSSION}

Close encounters between AGB stars and molecular clouds are apparently too infrequent to contribute primarily to the problem of silicon isotopic ratios in mainstream $\mathrm{SiC}$ grains. The results have shown that, for the most part, stars experience a small perturbation in their orbits and they are less likely to deviate largely from their initial circular orbits. Close to $40 \%$ of the stars suffered inward migration. Only a small fraction of AGB stars 1\% migrated to larger radii. Wielen et al. ${ }^{[13]}$ found that the Sun was born at galactocentric radius $\mathrm{R}=6.6 \mathrm{kpc}$ and has in $4.5 \mathrm{Gyr}$ diffused outwards to its present location at $\mathrm{R}=8.5 \mathrm{kpc}$. My calculations found in two billion years a percentage of $0.4 \%$ stars born between $4.0-4.5 \mathrm{kpc}$ migrate outward to 6.0-6.5 kpc zone where they give their AGB stardust to a presolar cloud. This result, in particular, is very interesting because Sellwood and Binney ${ }^{9}$ studied the scattering of stars by spiral waves and concluded that the grains must have migrated from $R \sim 4 \mathrm{kpc}$ to $~$ $6 \mathrm{kpc}$ where they were incorporated into a presolar cloud.

Migration of AGB stars from more central regions in the galaxy due to scattering by molecular clouds is deemed unlikely. The percentages the calculations have produced are small and cannot account for the observed richness in the heavier silicon isotopes when compared with to solar values. One hoped to see a larger fraction of stars scattered from their initial almost circular orbits to larger ones. I have shown only a few stars do that.

\section{ACKNOWLEDGEMENT}

The NASA Origin of Solar Systems Program has supported this study.

\section{REFERENCES}

1. Binney, J. and M. Merrifield, 1998. Galactic Astronomy.(Princeton, New Jersey.

2. Binney, J. and S. Tremaine, 1987. Galactic Dynamics. Princeton, New Jersey.

3. Clayton, D., 1997. Placing the sun and mainstream $\mathrm{SiC}$ particles in galactic chemo-dynamics evolution. Astrophys. J., 484: L67.

4. Hartmann, D., R. Epstein and S. Woosley, 1990. Galactic neutron stars and gamma-ray bursts. Astrophys. J., 348: 625.

5. Hoppe, P., S. Amari, E. Zinner, T. Ireland and R.S. Lewis, 1994. Carbon, nitrogen, magnesium, silicon and titanium isotopes compositions of single interstellar silicon carbide grains from the Murchison carbonaceous chondrite. Astrophys. J. 430: 870. 
6. Miyamoto, M. and R. Nagai, 1975. Threedimensional models for the distribution of mass in our Galaxy. Publ. Astron. Soc. Jap., 27: 431.

7. Press, V., S. Teukolsky, W. Vetterling and B. Flannery, 1992. Numerical Recipes in Fortran. Cambridge University Press, New York.

8. Pudritz, R.E., 2002. Clustered star formation and the origin of stellar masses. Science, 295: 68.

9. Sellwood, J.A. and J. Binney, 2001. Radial mixing in galactic discs. Monthly Notices R. Astronom. Soc., 336: 785 .

10. Solomon, P.M. and D.B. Sanders, 1985. Star formation in a galactic context: The location and properties of molecular clouds. Protostars and Planets II. University of Arizona Press, Tucson.

11. Tayler, R., 1996. Galaxies: Structure and Evolution. University Press, Cambridge.

12. Ward-Thompson, D., 2002. Isolated star formation: From cloud formation to core collapse. Science, 295: 76.

13. Wielen, R., B. Fuchs and C. Dettbarn, 1996. On the birth-place of the sun and the places of formation of other nearby stars. Astronom. Astrophys., 314: 438.
14. Zinner, E., L. Nittler, R. Gallino, A. Karakas, M. Lugaro, O. Straniero and J. Lattanzio, 2006. Silicon and carbon isotopic ratios in AGB stars: $\mathrm{SiC}$ grain data, models and the galactic evolution of the si isotopes. Astrophys. J., 650: 350.

15. Zwitter, T., A. Siebert, U. Munari, K. Freeman, C.A . Siviero, F.G. Watson, J.P. Fulbright, R.F.G. Wyse, R. Campbell, G.M. Seabroke, M. Williams, M. Steinmetz, O. Bienaymé, G. Gilmore, E.K. Grebel, A. Helmi, J.F. Navarro, B. Anguiano, C. Boeche, D. Burton, P. Cass, J. Dawe, K. Fiegert, M. Hartley, K. Russell, L. Veltz, J. Bailin, J. Binney, J. Bland-Hawthorn, A. Brown, W. Dehnen, N.W. Evans, P.R. Fiorentin, M. Fiorucci, O. Gerhard, B. Gibson, A. Kelz, K. Kujken, G. Matijevič, I. Minchev, Q.A. Parker, J. Peñarrubia, A. Quillen, M.A. Read, W. Reid, S. Roeser, G. Ruchti, R.D. Scholz, M.C. Smith, R. Sordo, E. Tolstoi, L. Tomasella, S. Vidrih and E. Wylie-de Boer, 2008. The radial velocity experiment (rave): Second data release. Astronom. J., 136: 421. 